\title{
Chaotic orbits obeying one isolating integral in a four-dimensional map
}

\author{
J. C. Muzzio ${ }^{1,2 \star}$ \\ ${ }^{1}$ Facultad de Ciencias Astronómicas y Geofísicas, Universidad Nacional de La Plata, Paseo del Bosque, 1900 La Plata, Argentina \\ ${ }^{2}$ Instituto de Astrofísica de La Plata (CCT CONICET La Plata-UNLP), Paseo del Bosque, 1900 La Plata, Argentina
}

Accepted 2017 October 9. Received 2017 October 9; in original form 2017 August 10

\begin{abstract}
We have recently presented strong evidence that chaotic orbits that obey one isolating integral besides energy exist in a toy Hamiltonian model with three degrees of freedom and are bounded by regular orbits that isolate them from the Arnold web. The interval covered by those numerical experiments was equivalent to about one million Hubble times in a galactic context. Here, we use a four-dimensional map to confirm our previous results and to extend that interval 50 times. We show that, at least within that interval, features found in lower dimension Hamiltonian systems and maps are also present in our study, e.g. within the phase space occupied by a chaotic orbit that obeys one integral there are subspaces where that orbit does not enter and are, instead, occupied by regular orbits that, if tori, bound other chaotic orbits obeying one integral and, if cantori, produce stickiness. We argue that the validity of our results might exceed the time intervals covered by the numerical experiments.
\end{abstract}

Key words: chaos - methods: numerical - celestial mechanics-galaxies: kinematics and dynamics.

\section{INTRODUCTION}

Autonomous Hamiltonian systems with three degrees of freedom can, in principle, support three types of orbits: regular that obey two isolating integrals besides energy, partially chaotic that obey only one integral besides energy and fully chaotic that obey just the energy integral. Nevertheless, it is still unclear whether partially chaotic orbits actually exist. While examples of those orbits have been presented by Contopoulos, Galgani \& Giorgilli (1978) and Pettini \& Vulpiani (1984), their existence has been denied by Froeschlé (1970), Froeschlé (1971) and Lichtenberg \& Lieberman (1992). Full details on this situation are given by Muzzio (2017), who also found partially chaotic orbits that are bounded by regular orbits in a toy model; the validity of his results is however limited to the time span covered by his numerical investigation that, although very long (about one million Hubble times if placed in a galactic context), is not infinite. As indicated by Muzzio (2017), if partially chaotic orbits actually exist, they can pose obstacles to chaotic diffusion: three-dimensional (3D hereafter) regular orbits cannot bound fully chaotic orbits that are 5D, but they can bound 4D partially chaotic orbits and these, in turn, can either bound or place obstacles to the fully chaotic orbits. It is thus important from a theoretical point of view to establish whether partially chaotic orbits exist or not.

Partially chaotic orbits are also of interest for dynamical astronomy. In our own work on the dynamics of triaxial stellar systems

^E-mail: jcmuzzio@fcaglp.unlp.edu.ar that goes from Muzzio (2003) to Carpintero \& Muzzio (2016, see the latter for references to other works), we have shown that partially chaotic orbits represent about 10 per cent of the orbits in those systems and have a distribution that differs from that of the fully chaotic orbits. Thus, it is necessary to distinguish partially from fully chaotic orbits in studies on the dynamics of elliptical galaxies. The presence of partially chaotic orbits in those systems had been noticed earlier by Goodman \& Schwarzschild (1981) and Merritt \& Valluri (1996), but no particular importance was given to them at that time. Besides, the phenomenon of 'stable chaos' investigated by Milani \& Nobili (1992) and Milani et al. (1997) might be an example of partially chaotic orbits in the Solar system.

It will be worthwhile, before going on, to clarify the meaning of some terms to avoid confusion. The terms partially and fully chaotic orbits were proposed by us (Muzzio, Carpintero \& Wachlin 2005) to design what Pettini \& Vulpiani (1984) had called, respectively, weakly and strongly chaotic orbits. The reason was that, as indicated by Contopoulos (2002), the terms weak and strong chaos are used by other authors (see, e.g. Voglis, Contopoulos \& Efthymiopoulos 1998) to refer to the value of the Lyapunov exponent (LE hereafter) and that is the meaning that we prefer for those terms. Therefore, what Pettini \& Vulpiani (1984) called weakly chaotic orbit is just the same of what we call partially chaotic orbit. What most authors nowadays call weakly chaotic orbit is an orbit with a low value of its largest LE (the only one that is usually computed). But a partially chaotic orbit is one that has its largest LE different from zero, no matter whether it is large or small, and its second largest LE equal to zero (see Section 2.2 for details). 
The case of sticky orbits is different. They are orbits that stay for a long time in a certain region of phase space and, then, diffuse into another region. One example is orbits that stay long around islands of stability, another is orbits close to the unstable asymptotic curves of unstable periodic orbits. They were first noted by Contopoulos (1971), and later on by Shirts \& Reinhardt (1982), Karney (1983) and Meiss et al. (1983); Karney seems to have been the first one to use the term sticky in this context. For reviews and more recent results, see Contopoulos \& Harsoula (2010a) and Contopoulos \& Harsoula (2010b). Sticky orbits play an important role in barred spiral galaxies because they support the shape of the bar, as well as the rings and spiral arms, for very long times before escaping through Arnold diffusion (see, e.g. Harsoula \& Kalapotharakos 2009; Contopoulos \& Harsoula 2013). For a recent and detailed study of the speed of that diffusion, see Efthymiopoulos \& Harsoula (2013). Stickiness is a phenomenon different from partial and full chaos and, in principle, it can affect both partially and fully chaotic orbits, although thus far there were no known examples because most authors do not make the distinction between partially and fully chaotic orbits in their studies. We will present here, however, an example of stickiness in a partially chaotic orbit (at least within the interval covered by our numerical iterations).

As explained by Muzzio (2017), it would be very difficult to extend further his investigation on a toy Hamiltonian model, but an excellent opportunity is offered instead by 4D maps. These maps are a prototype of the Poincaré map that one obtains with a cut through the phase space of an autonomous Hamiltonian system with three degrees of freedom and they do not demand the numerical integration of differential equations. In fact, the results of Froeschlé (1971) were obtained with a map, a pioneering study that he continued in Froeschlé (1972), Froeschlé \& Scheidecker (1973a) and Froeschlé \& Scheidecker (1973b). But, excellent as it is, his work was limited by the computational means available at that time and, in particular, it did not reach the degree of resolution needed to find the very fine regions where Muzzio (2017) showed that may lurk the partially chaotic orbits. Therefore, we decided, resorting to the means nowadays available, to investigate the possible existence of partially chaotic orbits in the same map he had studied and that is the subject of this paper. Besides providing strong evidence that partially chaotic orbits exist in the map studied by Froeschle, the present investigation extends that of Muzzio (2017) in two ways. First, it yields similar results using a map instead of a Hamiltonian model and, secondly, it extends the validity of those results (limited to the interval covered by the numerical integrations or iterations) to a 50 times longer interval. We used the same techniques we had employed in our investigation of the toy model, adapting them to the case of maps. We also made good use of 3D plots using colour as the fourth dimension, a technique developed by Patsis \& Zachilas (1994) to investigate Hamiltonian systems with three degrees of freedom (see also Katsanikas, Patsis \& Contopoulos 2013, for a more recent application to a case that includes stickiness). A similar technique was applied by Richter et al. (2014) to the case of maps.

The organization of this paper is very similar to that of our previous one. The following section describes the map and the techniques we used to study its orbits. The results of a search for possible partially chaotic orbits are presented in Section 3, where we also isolate one of them using the integral it obeys. In Section 4, we present the regular orbits that bound that partially chaotic orbit, and show 3D Poincare maps with those three orbits plus a few other interesting ones and, finally, we explain our conclusions in Section 5.

\section{MAP AND NUMERICAL METHODS}

\subsection{The map}

We chose the 4D map:

$x_{i+1}=x_{i}+a_{1} \sin \left(x_{i}+y_{i}\right)+b \sin \left(x_{i}+y_{i}+z_{i}+t_{i}\right)$

$y_{i+1}=x_{i}+y_{i}$

$z_{i+1}=z_{i}+a_{2} \sin \left(z_{i}+t_{i}\right)+b \sin \left(x_{i}+y_{i}+z_{i}+t_{i}\right)$

$t_{i+1}=z_{i}+t_{i}$,

where the values of $x, y, z$ and $t$ lie always between $-\pi$ and $+\pi$ and the determinant of its Jacobian matrix is equal to 1 . As indicated in the introduction, this model was extensively investigated by Froeschlé (1971), Froeschlé (1972), Froeschlé \& Scheidecker (1973a) and Froeschlé \& Scheidecker (1973b). For the present investigation, we adopted $a_{1}=a_{2}=-0.25$ and $b=0.02$ which yield plots similar to those of the double resonance studied by Muzzio (2017).

\subsection{Numerical methods}

We explored the phase space of our model using orbits with initial coordinates $z=t=0$ and different $x$ and $y$ values. To follow each orbit and at the same time compute the four LEs, we adapted for our map the LIAMAG routine, kindly provided by D. Pfenniger (see Udry \& Pfenniger 1988) and originally written for orbits in a Hamiltonian system. This was simply done replacing the call to the Runge-Kutta-Fehlberg subroutine that integrated the orbits and the variational equations by the map equations (1) through (4) and by the Jacobian matrix of the map. We prepared two versions of the routine, one using double precision as the original version, and a second one using quadruple precision. The latter allowed us to follow the orbits with much longer iterations but, of course, it runs much slower.

The LEs $\lambda_{1}>\lambda_{2}>\lambda_{3}>\lambda_{4}$ have the property that $\lambda_{i}=-\lambda_{5-i}$, because the determinant of the Jacobian matrix is equal to 1 . Besides, each isolating integral makes one $\lambda_{i}=-\lambda_{5-i}$ pair zero so that, considering only the two largest LEs, we have that both are zero for regular orbits, only one is zero for partially chaotic orbits, and none is zero for fully chaotic orbits. Nevertheless, since the number of iterations computed for the map is necessarily finite, numerical LEs can tend towards zero as the number of iterations in the map increases, but they remain always larger than a limiting value that can be estimated to be of the order of $\ln N / N$, where $N$ is the number of iterations. This is a coarse estimate only, and the limiting value should be determined in every case (see, e.g. Zorzi \& Muzzio 2012, for details).

Fig. 1 gives the evolution of the LEs of three different orbits as the number of iterations increases. For each orbit, full lines were used for $\lambda_{1}$ and dots for $\lambda_{2}$. The regular orbit r1 (see Section 4.1) is shown in black, the partially chaotic orbit pch (see Section 3.1) in red in the electronic version and the fully chaotic orbit with initial conditions $x=0.87500, y=2.015625, z=0, t=0$ in green in the electronic version. We notice that, for the regular orbit, both LEs decrease almost linearly with the number of iterations in this double logarithmic plot, and that the same happens with the $\lambda_{2}$ value of the 


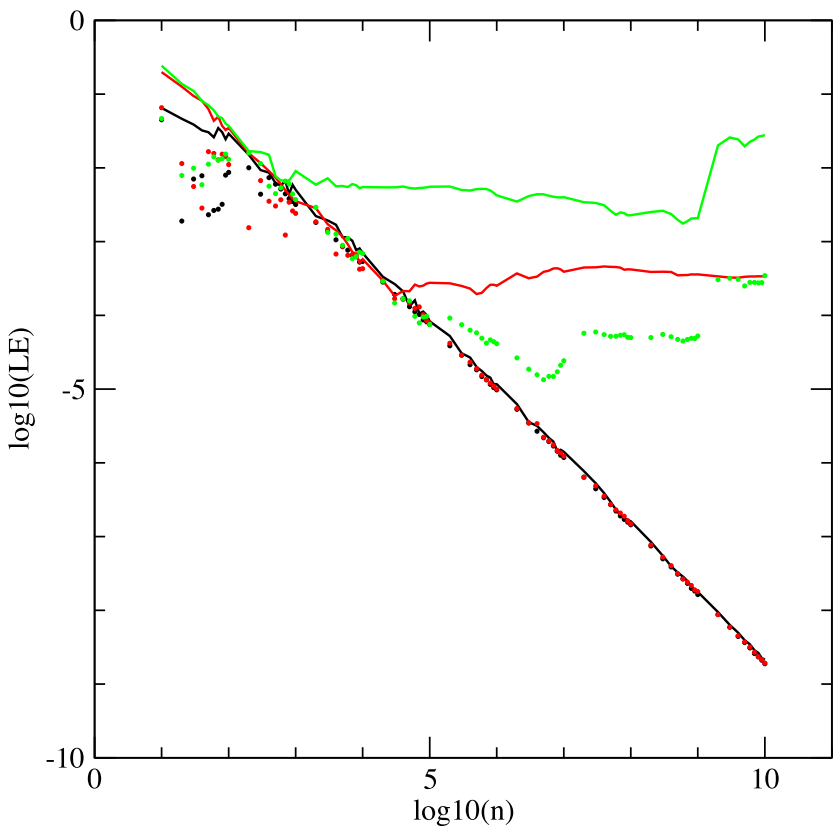

Figure 1. Evolution of the LEs with the number of iterations for regular, partially chaotic (red in the electronic version) and fully chaotic (green in the electronic version) orbits. For each orbit, $\lambda_{1}$ is shown with full lines and $\lambda_{2}$ with dots.

partially chaotic orbit. The $\lambda_{1}$ value of the partially chaotic orbit, as well as both LEs of the fully chaotic orbit, instead, clearly do not go to zero as the number of iterations increases.

The longest computations done with the double precision routine reached $10^{8}$ iterations and a comparison with the results of the quadruple precision routine showed that the errors in $x, y, z$ and $t$ were at most of the order of $10^{-5}$. With the quadruple precision routine, we reached up to $10^{10}$ iterations and a comparison with results obtained reversing the iteration and using the inverse map suggested that the errors in $x, y, z$ and $t$ were at most of the order of $2 \times 10^{-7}$. Taking our map as a Poincaré map, each iteration in the former corresponds to the interval elapsed between two cuts to obtain the latter, i.e. we can take an iteration as the characteristic time of the orbits in the Hamiltonian which corresponds to the Poincaré map. Thus, our $10^{10}$ iterations can be taken as covering an interval of $10^{10}$ characteristic times and, since 1 Hubble time corresponds to about 200 characteristic times for the elliptical galaxies we investigated previously (see, e.g. Zorzi \& Muzzio 2012), that is equivalent to about $5 \times 10^{7}$ Hubble times in a galactic context.

Orbits that obey no integral fill in a $4 \mathrm{D}(x, y, z, t)$ space and those that obey one integral, $I_{1}(x, y, z, t)=C_{1}=$ constant, occupy a 3D space because, in principle, we might put, e.g. $t$ as a function of $x$, $y, z$ and $C_{1}$. Besides, orbits that also obey a second integral, $I_{2}(x, y$, $z, t)=C_{2}=$ constant, occupy a $2 \mathrm{D}$ space because, in principle, we might also put, e.g. $z$ as a function of $x, y, C_{1}$ and $C_{2}$. Thus, in order to recognize regular from chaotic orbits we need to take a cut, say, $z=z_{\mathrm{o}}$ to get curves for the regular orbits and surfaces for the chaotic ones. In practice, the cut has to be replaced by a slice $z \simeq z_{0}$ to get a reasonable number of points, but the width of the slice can be kept thin enough to avoid affecting the recognition of the orbits. It is important to remember that, as indicated by Muzzio (2017), these surfaces and curves do not lie on a plane but are warped because they are embedded in a 3D space. A second slice, say $t \simeq t_{0}$, is needed to distinguish partially from fully chaotic orbits; the former will appear as curves and the latter as surfaces (and regular orbits as points)

Therefore, we prepared a quadruple precision program that gave the successive iterations of the map (1) through (4) and took slices $z \simeq z_{\mathrm{o}}$ and $t \simeq t_{\mathrm{0}}$. The results presented here were obtained with $10^{10}$ iterations and a comparison with results obtained reversing the iteration and using the inverse map suggested that the errors in $x$, $y, z$ and $t$ were at most of the order of $2.5 \times 10^{-7}$, i.e. essentially the same as those of the LIAMAG routine for the same number of iterations, as could be expected. As explained before, our $10^{10}$ iterations correspond to about $5 \times 10^{7}$ Hubble times in a galactic context.

In order to get a clear view of orbital structures at several stages of our investigation, we found very useful the technique of Patsis $\&$ Zachilas (1994) who used 3D plots plus colour to represent the fourth dimension. We have adopted their method using gnuplot (Copyright (C) 1986-1993, 1998, 2004, 2007 Thomas Williams, Colin Kelley) to make the plots.

To represent the warped surfaces and curves that result from our cuts, we resorted to the method of Muzzio (2017) that used Fourier series to fit a chosen regular orbit and to refer to it the results from nearby orbits. Here, we adopted the $x, y$ and $t$ variables resulting from the $|z| \leq 10^{-6}$ slice and, for an orbit selected as reference, we normalized each one of those variables subtracting the corresponding value for the centre of the orbit and dividing the result by the dispersion of the variable in question. Then, we transformed that normalized coordinate system into a cylindrical one and, using the azimuth angle $\phi$ as argument, we obtained the best-fitting Fourier series for the radius $R$ and the vertical distance $Z$ (notice that this new $Z$ variable is actually the normalized value of the original $t$, not $z$, variable). For nearby orbits, we obtained the differences between the values of their variables (normalized using the same centre and dispersions of the orbit taken as reference) and those given by the corresponding Fourier series, so that we can travel along the orbits, following $\phi$, and the differences between their $R$ and $Z$ values and the corresponding ones of the orbit taken as reference can be plotted with considerable details. Here, we have adopted a cylindrical system of coordinates, rather than the spherical one we used in our previous work, because the former is better to show the warped surfaces we found in the present investigation.

We experimented with different numbers of terms and found that the mean square error decreased as we increased that number up to about 651 terms [that is, up to terms $\sin (325 \phi)$ and $\cos (325 \phi)$ ] and reached a plateau where increasing the number of terms did not significantly decreased the mean square error any further, so that we adopted that number of terms for our computations. For slices with $|z| \leq 10^{-6}$, the resulting mean square errors of the $x, y$ and $t$ variables turned out to be of the order of $0.15 \times 10^{-7}$, $0.74 \times 10^{-7}$ and $6.4 \times 10^{-7}$, respectively. For slices with double and half widths, the errors were proportional to the width of the slice, as could be expected. To estimate the numerical errors of iteration we obtained, the Fourier series using only the first 20 per cent points and computed the mean square errors of the last 20 per cent points with respect to those series. The dispersions turned out to be essentially the same, so that the errors of the numerical iteration should be much smaller than the dispersion caused by the slice widths.

\section{PARTIALLY CHAOTIC ORBITS}

The first step of our investigation was to search for possible partially chaotic orbits in our map. We performed that search using LEs but, as indicated by Lichtenberg \& Lieberman (1992), we can never 


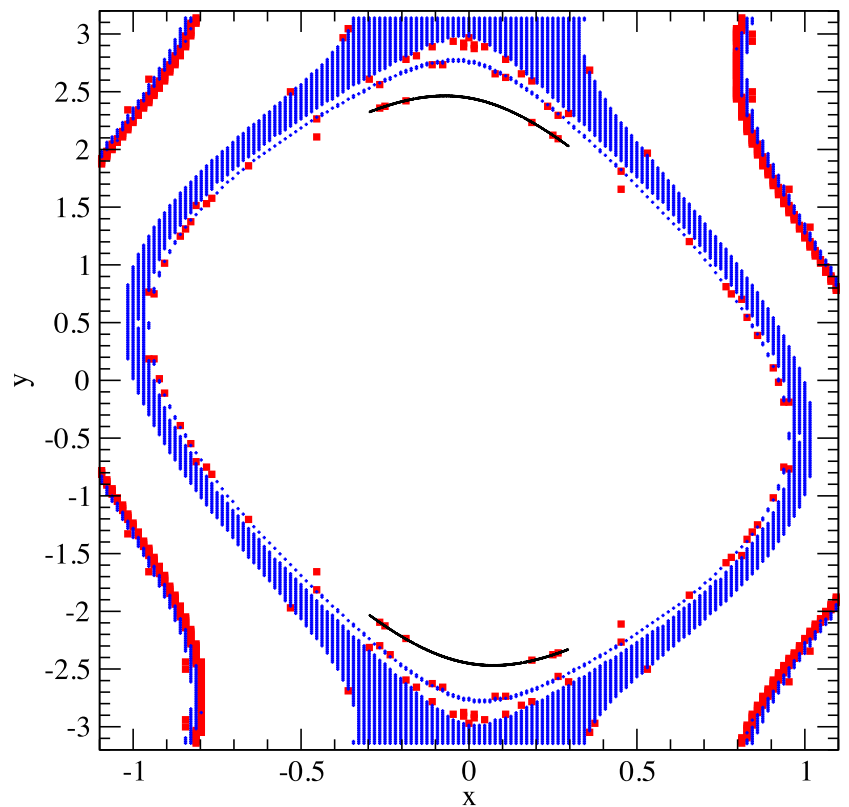

Figure 2. Initial conditions on the $(x, y)$ plane of orbits classified as regular, partially and fully chaotic from the values of their LEs. The blank areas correspond to regular orbits, partially chaotic orbits are shown as filled squares (red in the electronic version) and fully chaotic orbits as plus signs (blue in the electronic version). The small dots that trace the two curves above and below were obtained taking two slices $|z| \leq 5 . \times 10^{-5}$ and $|t| \leq$ 5. $\times 10^{-5}$ from the partially chaotic orbit pch (see Section 3.2 for details).

be sure that the partially chaotic orbits found in that way will not appear as fully chaotic with LEs computed with a larger number of iterations. Thus, it should be recalled that the orbits that we will refer to as partially chaotic here can be regarded as such only over the span covered by our iterations.

\subsection{The search}

We began our search preparing a sample of initial conditions with $z=t=0$ and a grid of $x$ and $y$ values with $-\pi<x<\pi$ and $-\pi<y<\pi$ and $2^{-6}=0.015625$ spacing. The advantage of taking these initial conditions is that the plots obtained with them will be useful as comparison when, later on, we will take slices with $z \simeq 0$ and $t \simeq 0$. Using those initial conditions, we computed the orbits over $10^{7}$ iterations and obtained the LEs which, in turn, we used to classify the orbits as regular, partially or fully chaotic. Fig. 2 is a $(x, y)$ plot where the blank areas correspond to initial conditions that yielded regular orbits, while those that yielded partially and fully chaotic orbits are shown, respectively, as filled squares (red in the electronic version) and plus signs (blue in the electronic version). The chains of small dots resulted from taking two slices $|z| \leq 5 . \times 10^{-5}$ and $|t| \leq 5 . \times 10^{-5}$ from a partially chaotic orbit, and will be explained in Section 3.2. The figure has several features in common with fig. 1 of Muzzio (2017); we notice a central region dominated by regular orbits, surrounded by another one dominated by fully chaotic ones, with most of the partially chaotic orbits lying on the border between those regions.

What most interests us here is that, as in our previous work, several partially chaotic orbits can also be found well inside the regular domain. Therefore, we made a higher resolution plot of the region $-0.265<x<-0.250,-2.125<y<-2.100$, where a couple of partially chaotic orbits can be seen in Fig. 2, which

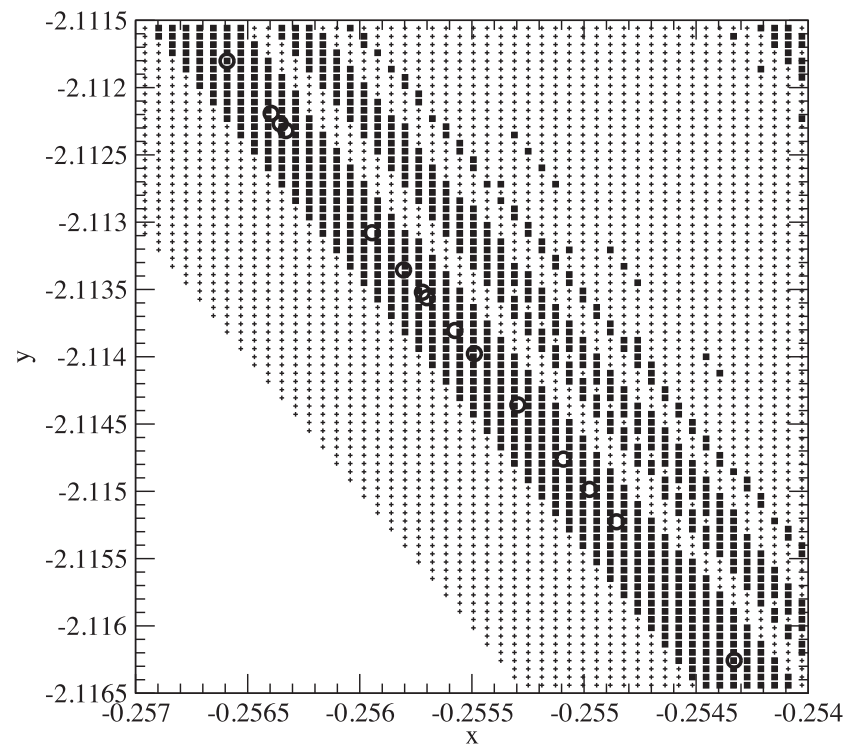

Figure 3. High-resolution plot of a small section of Fig. 1. Regular orbits are shown as crosses and partially chaotic orbits as filled squares. The open circles result from taking two slices $|z| \leq 5 . \times 10^{-5}$ and $|t| \leq 5 . \times 10^{-5}$ from the same partially chaotic orbit of Fig. 2. The white area was not investigated with this high resolution, but lower resolution plots showed only regular orbits there.

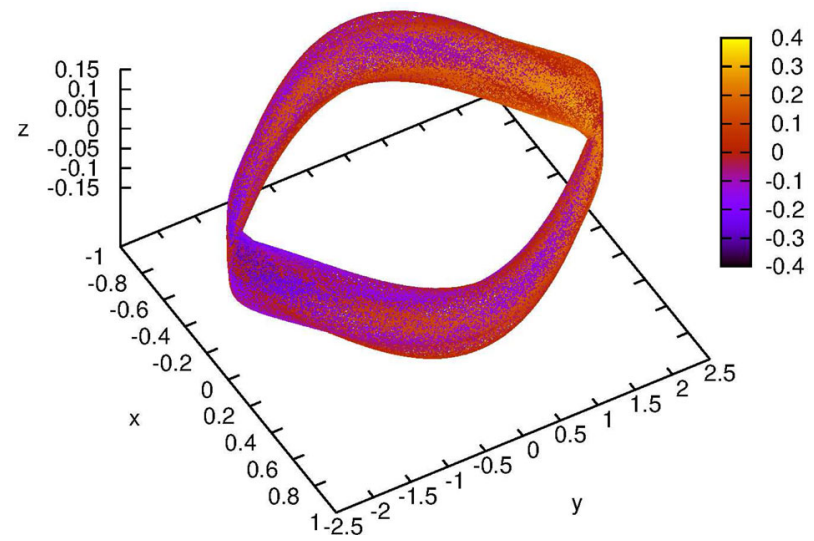

Figure 4. Partially chaotic orbit pch from the chain shown in Fig. 2. It is shown in the 3D space $(x, y, z)$ with the fourth dimension $t$ given by the colour scale in the electronic version.

showed a continuous chain of partially chaotic orbits as we had found before. We did plots of increasingly higher resolution that showed the same and, besides, resolved the chain in several parallel chains of partially chaotic orbits separated by similar chains of regular orbits. The plots were obtained with $10^{8}$ iterations, but the regular and partially chaotic nature of several of the orbits was confirmed running the LIAMAG routine up to $10^{10}$ iterations. Fig. 3 shows a small part of one of our plots obtained with a grid spacing of $2^{-14} \simeq 0.000061035$. Regular orbits are shown as crosses and partially chaotic orbits as filled squares. We also show, as open circles, the results from taking two slices $|z| \leq 5 . \times 10^{-5}$ and $|t|$ $\leq 5$. $\times 10^{-5}$ from a partially chaotic orbit that will be explained in Section 3.2.

Fig. 4 shows, in the 3D space $(x, y, z)$ and using colour to represent the fourth dimension $t$, the orbit whose initial conditions are $x=-0.256591796875, y=-2.11180224609375, z=0, t=0$ 


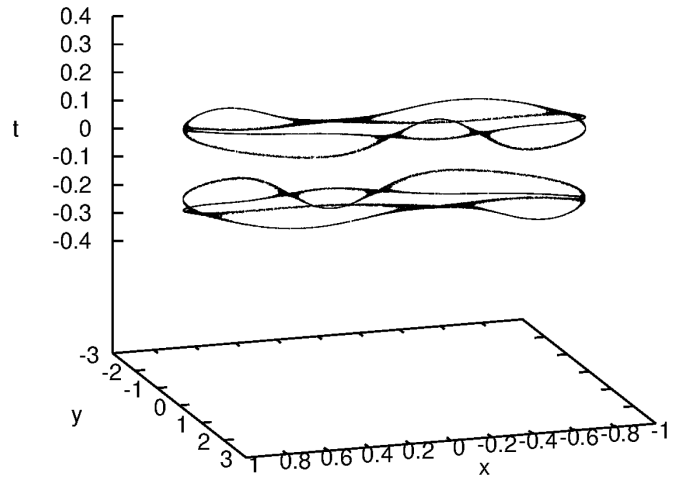

Figure 5. A slice $|z| \leq 10^{-6}$ of the partially chaotic orbit pch from Figs 3 and 4 in the 3D space $(x, y, t)$.

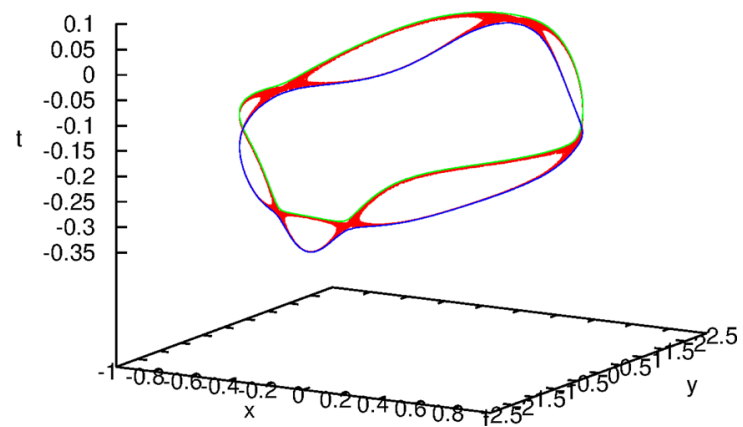

Figure 6. A slice $|z| \leq 10^{-6}$ of the partially chaotic orbit pch from Figs 3 and 4 in the 3D space ( $x, y, t$, red in the electronic version) together with the same slices from the two nearby regular orbits $\mathrm{r} 1$ (green in the electronic version) and $\mathrm{r} 2$ (blue in the electronic version). The slice has actually two parts as shown in Fig. 4 but, for clarity, only the part with lower $t$ values is shown here.

and that we will dub pch hereafter. This is one of the partially chaotic orbits that lie on the chain shown in Fig. 3 and its partially chaotic nature was confirmed by the LEs obtained running the LIAMAG for $10^{10}$ iterations. It has the form of a torus and some mixing of the colours might be present, a characteristic of chaotic orbits in this sort of plot as indicated by Patsis \& Zachilas (1994), but if it exists it is far from clear. In fact, except perhaps for the colour distribution, similar plots for other orbits from the same region, either regular or partially chaotic, look very much the same.

Fig. 5 shows a slice $|z| \leq 10^{-6}$ from the orbit of Fig. 4 in the $3 \mathrm{D}$ space $(x, y, t)$ and we see that it has two parts, very similar to each other, one with mainly positive $t$ values and another with mainly negative $t$ values. For the time being, we will concentrate on the part that corresponds to the lower values of $t$ which are shown again in Fig. 6. ${ }^{1}$ The figure shows the aforementioned slice from the orbit pch in the 3D space $(x, y, t$, red in the electronic version) together with similar slices from regular orbits $r 1$ (green in the electronic version) and r2 (blue in the electronic version) that will be explained in Section 4.1. As anticipated, the points lie on a warped surface (actually, it has a very small width because there is a finite range of $z$ values) and not on a plane. The regular orbits are curves that bound the surface occupied by the chaotic orbit, which is a double orbit (with each part similar to each one of the regular

\footnotetext{
${ }^{1}$ Notice that Figs 5 and 6 show the 3D space from two different points of view and that is why, in the latter, the orbits seem to reach positive $t$ values, it is just an effect of perspective.
}

orbits) linked by a bifurcation that is the most likely source of its chaos. All this is very similar to what we found in Muzzio (2017).

\subsection{The integral of motion of partially chaotic orbits}

Since the initial conditions we used to obtain Figs 2 and 3 had all $z=t=0$, these figures are similar to Poincaré maps resulting from two cuts $z=0$ and $t=0$. But, for a true Poincaré map, we should have selected orbits that have the same value of the integral that obey the partially chaotic orbits (and one of the two integrals that obey the regular orbits), so as to get $1 \mathrm{D}$ curves for the partially chaotic orbits and points for the regular ones. Therefore, the lane of partially chaotic orbits in Fig. 3 can be seen as the surface that results from placing one beside another the curves corresponding to partially chaotic orbits with different values of that integral, again a situation very similar to the one found in Muzzio (2017), and our problem is to segregate the different curves that make up the lane. But here we have the enormous advantage that we can obtain many more points per orbit than we had in our previous work, so that it is perfectly possible to make a long iteration of a partially chaotic orbit and to take slices $z \simeq 0$ and $t \simeq 0$ thin enough to get the curve it traces on the $(x, y)$ plane.

We selected the partially chaotic orbit pch, iterated it $10^{10}$ times and obtained the slices $|z| \leq 5 . \times 10^{-5}$ and $|t| \leq 5 . \times 10^{-5}$. The result is shown in Figs 2 and 3 where we notice that we obtained a curve that is very thin indeed confirming that it corresponds to a partially chaotic orbit as we had already found with the LEs. One minor difference with the result obtained by Muzzio (2017) is that here the curves corresponding to constant values of the integral run parallel to the lane, while in the toy Hamiltonian model they crossed it (cf. fig. 8 of our previous paper).

\section{BOUNDING REGULAR ORBITS}

\subsection{Finding the boundaries}

The task of finding regular orbits that obey the same integral as partially chaotic orbit pch and bound it is also greatly simplified thanks to the large number of points that we have at our disposal here. We can simply extrapolate the lines obtained from the two slices in $z$ and in $t$, rather than having to resort to surfaces as done by Muzzio (2017). Extrapolations are always risky and non-linear extrapolations are the riskiest, so that we decided to take only a small section from the right tip of the lower curve of Fig. 2 given by the slices $|z| \leq 5 . \times 10^{-5}$ and $|t| \leq 5 . \times 10^{-5}$ and to perform a linear extrapolation. Of course, as the tori of the regular orbits fit one inside the other, one can choose from the extrapolation many points that correspond to different tori that share the same value of the integral with the partially chaotic orbit pch. We chose one with $x=0.30250000, y=-2.31987691, z=0.0, t=0.0$ that, without demanding much extrapolation, provides the initial conditions of the regular orbit we dubbed $\mathrm{r} 1$ that yields clear plots. The dispersion of the points around the extrapolating straight line was $2.5 \times 10^{-6}$, further proof that we are dealing with a very thin line, and the estimated error of the $y$ value of the extrapolated point was $5.0 \times 10^{-6}$. To get an estimate of how this result is affected by the fact that the line is not perfectly straight, we fitted one line to the first half of points and another to the second half and the $y$ difference between the two extrapolations at $x=0.30250000$ turned out to be $6.6 \times 10^{-5}$, a precision more than enough for our purposes.

The slices $|z| \leq 5 . \times 10^{-5}$ and $|t| \leq 5 . \times 10^{-5}$ from orbit $\mathrm{r} 1$ produce points on the extrapolation of the left tip of the same curve, 
so that it cannot be used to find the other bounding orbit as we had expected. The reason is that this second bounding orbit has no points near $z=t=0$, but this problem was easily solved taking from the partially chaotic orbit pch two new slices $|z| \leq 5 . \times 10^{-5}$ and $|t+0.265| \leq 5 . \times 10^{-5}$. Another linear fit and extrapolation to the tip of the resulting curve let us find the initial conditions for the regular orbit that we dubbed r2 at $x=0.17220000, y=-2.39949868$, $z=0.0, t=-0.265$.

Fig. 6 shows that, indeed, partially chaotic orbit pch is bounded by the regular orbits $\mathrm{r} 1$ and $\mathrm{r} 2$. But a clearer view can be obtained with the technique developed by Muzzio (2017) to get 3D Poinceré maps that offers 2D plots rather than the 3D one shown in the figure, and that will be the subject of the next section.

The surface that results from taking the slice $|z| \leq 5 . \times 10^{-5}$ from the orbit pch has holes in it, as can be seen in Fig. 6, so that we also searched for orbits inside those holes to include them in our 3D Poincaré maps. Taking from orbit pch two new slices $|z|$ $\leq 5 . \times 10^{-5}$ and $|t+0.230| \leq 5 . \times 10^{-5}$ and performing another linear extrapolation, we found the partially chaotic orbit phol and the regular orbit rhol whose initial conditions are, respectively, $x=0.26465000, y=-2.34147742, z=0.0, t=-0.230$ and $x=0.26342500, y=-2.34259592, z=0.0, t=-0.230$.

The regular or partially chaotic nature of all the orbits found in the present subsection was confirmed with runs of $10^{10}$ iterations with the LIAMAG routine.

\subsection{D Poincaré maps}

We chose the part with lower $t$ values that results from taking the slice $|z| \leq 10^{-6}$ from orbit $\mathrm{r} 1$ as our reference orbit and we computed the mean values $\left(<x>,<y>\right.$ and $<t>$ ) and the dispersions $\left(\sigma_{x}, \sigma_{y}\right.$ and $\sigma_{t}$ ) of its $x, y$ and $t$ values. Taking those mean values as the centre of the orbit, we computed the normalized values $(x-<x>) / \sigma_{x}$, $(y-<y>) / \sigma_{y}$ and $(t-<t>) / \sigma_{t}$, and used these normalized values to define a new cylindrical system of coordinates, with azimuth angle $\phi$, radius $R$ and vertical distance $Z$ (recall that this $Z$ is just the normalized value of $t$ and has nothing to do with $z$ ). Then, taking $\phi$ as argument, we adjusted each normalized coordinate with a Fourier series and we used them to iteratively improve the centre of the orbit. Finally, we obtained new Fourier series to represent $R$ and $Z$ as functions of $\phi$. The differences between the true values and those given by the series, i.e. the residuals, were used to represent orbit $\mathrm{r} 1$ in our 3D Poincaré maps, i.e. straight lines with some dispersion through $R=0$ and $Z=0$, respectively.

For other orbits, we normalized their $x, y$ and $t$ values using the same centre and dispersions adopted for the r1 orbit and obtained the corresponding $\phi, R$ and $Z$ values. Finally, using their $\phi$ values as argument of the Fourier series obtained for $r 1$, we obtained the differences between their $R$ and $Z$ values and those given by the series. In other words, our 3D Poincaré maps are just the differences between each orbit and $\mathrm{r}$, so that we can clearly represent those small differences as we follow the orbit through all the different azimuth angles.

Fig. 7 presents the result and, since the slices are warped, there seems to be some crossing among the different orbits, but this is only apparent. The $\phi$ versus $R$ plot (above) clearly shows that the regular orbits bound the partially chaotic orbits everywhere except for $R$ values very close to zero but, at the same time, the $\phi$ versus $Z$ plot (below) clearly shows that the regular orbits bound the partially chaotic orbit for the corresponding $Z$ values. That is the crossing of orbits takes place at different values of $\phi$ in each plot, so that there is no actual crossing in $3 \mathrm{D}$ space.
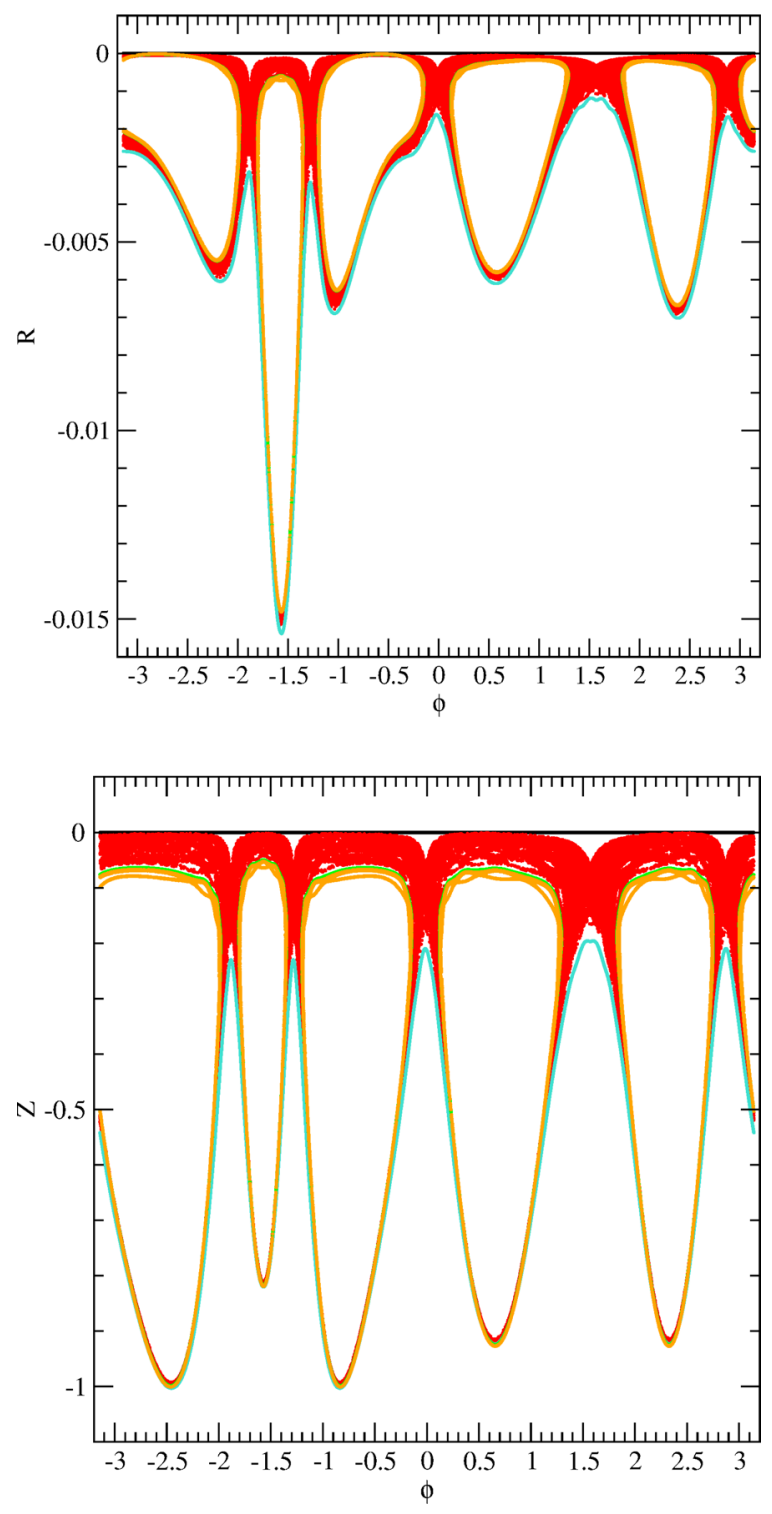

Figure 7. 3D Poincaré maps ( $\phi, R$, above) and ( $\phi, Z$, below) of orbits $\mathrm{r} 1, \mathrm{r} 2$ (turquoise in the electronic version), pch (red in the electronic version), rhol (green in the electronic version) and phol (orange in the electronic version). They correspond to the $|z| \leq 10^{-6}$ slice and to the part of the orbits with lower $t$ values. The ordinates give the differences between the values of $R$ and $Z$, respectively, of each orbit and those given by the Fourier series fitted to the corresponding values of orbit $\mathrm{r} 1$. See the text for explanation.

Of particular interest is the fact that inside partially chaotic orbit pch, and separated from it by regular orbit rhol, lies partially chaotic orbit phol. Therefore, we not only have the partially chaotic orbit pch well isolated from the rest of the phase space by regular orbits $\mathrm{r} 1$ and $\mathrm{r}$, but it even has inside it the partially chaotic orbit phol well protected by the cocoon provided by regular orbit rhol. As could be expected, the largest LE of orbit phol is lower $\left(3.5 \times 10^{-5}\right)$ than that of orbit pch $\left(3.4 \times 10^{-4}\right)$; as a comparison, for $10^{10}$ iterations, the LEs of regular orbits, and also the lowest LE of those partially chaotic orbits, are about $2 . \times 10^{-9}$.

Fig. 8 is like Fig. 7, but for the part of the orbits with higher $t$ values. Although we had not used that part of orbit pch to find the bounding regular orbits and those inside its holes, this new figure 

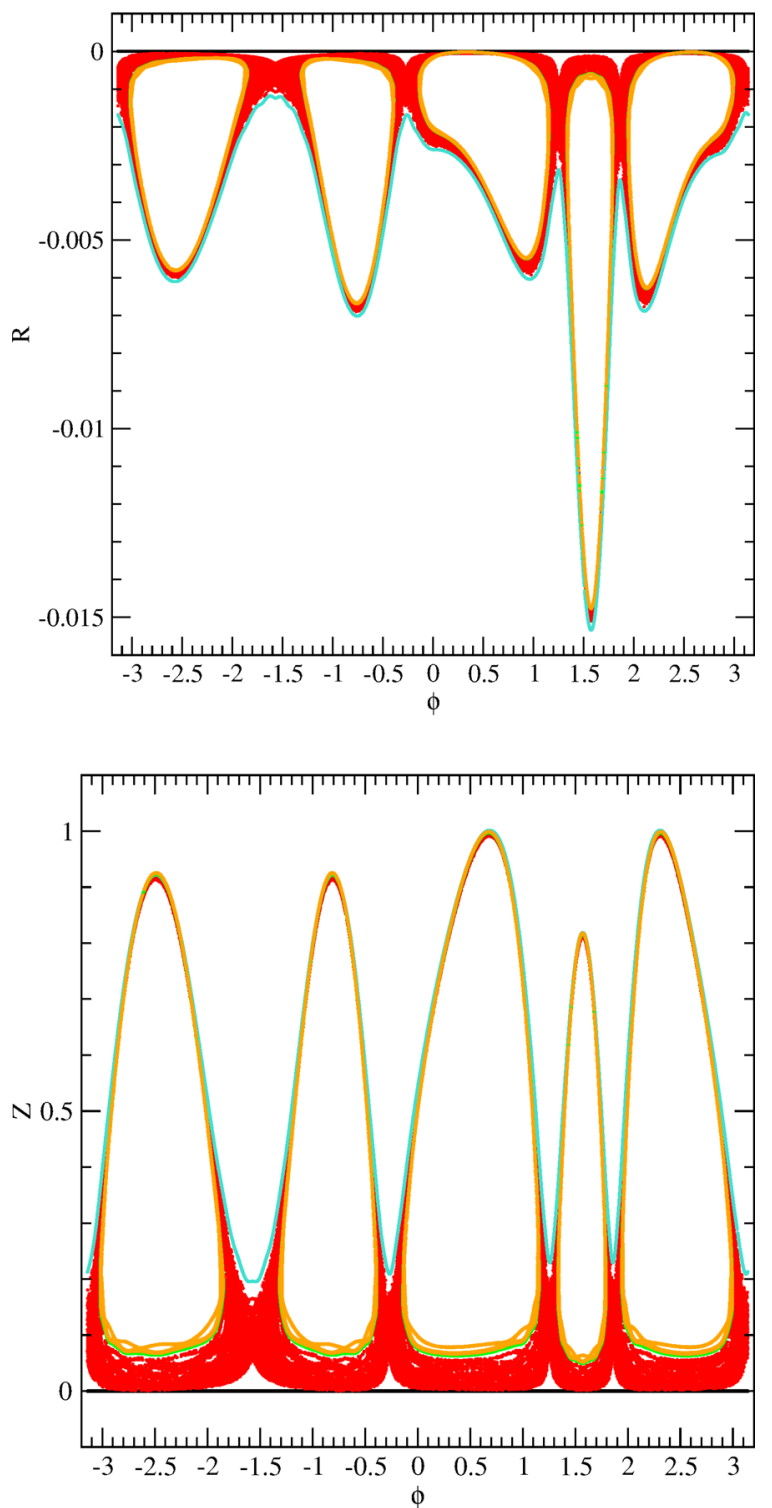

Figure 8. Same as Fig. 7, but for the part of the orbits with higher $t$ values.

tells the same story as the previous one: partially chaotic orbit pch is bounded by regular orbits $\mathrm{r} 1$ and $\mathrm{r} 2$ and contains inside its hole partially chaotic orbit phol separated from pch by regular orbit rhol.

\subsection{Stickiness}

When we were looking for the bounding regular orbits, we performed a few experiments fitting planes to small sections of the $|z|$ $\leq 10^{-5}$ slice of orbit pch. It was soon clear that the extrapolations done in that way could not reach distances as long as those obtained fitting a line to the slices $|z| \leq 5 . \times 10^{-5}$ and $|t| \leq 5 . \times 10^{-5}$ and that was the method adopted here. Nevertheless, in the process we found the partially chaotic orbits pchb $(x=0.26269580$, $y=-2.34256250, z=0.0, t=-0.23280000)$ and pchc $(x=0.26313362, y=-2.34209380, z=0.0, t=-0.23280000)$ and the regular orbit cant $(x=0.262461560, y=-2.34271880$, $z=0.0, t=-0.23280000)$. Their regular or partially chaotic nature was confirmed running the LIAMAG routine for $10^{10}$ iterations. Fig. 9 shows on the $(x, y)$ plane the slice $|z| \leq 10^{-5}$ of orbits pch, pchb

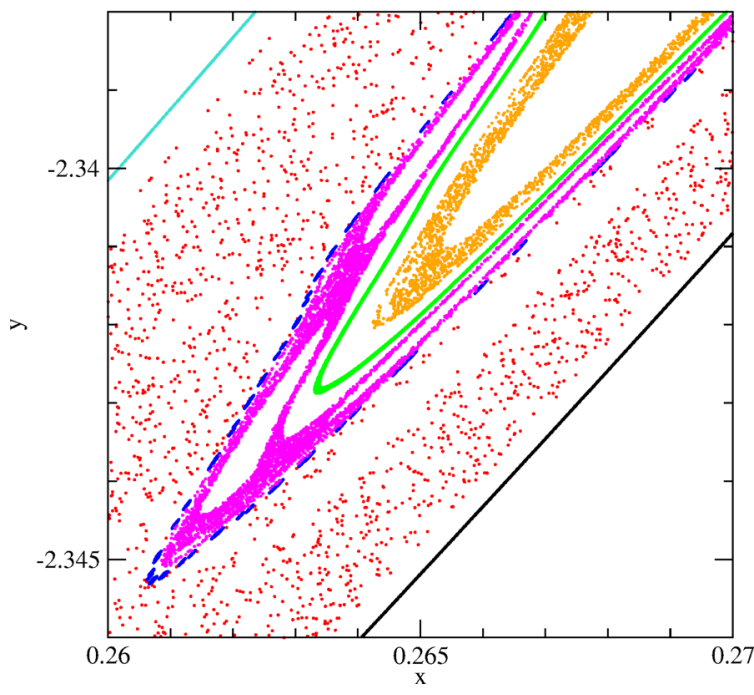

Figure 9. $x$ versus $y$ plot of the slice $|z| \leq 10^{-5}$ showing part of the hole in the orbit pch (red in the electronic version), together with orbits $r 1, r 2$ (turquoise in the electronic version), rhol (green in the electronic version), phol (orange in the electronic version), cant (blue in the electronic version) and pchb (magenta in the electronic version).

and cant (the same slice for orbits $\mathrm{r} 1, \mathrm{r} 2$, rhol and phol were also added for comparison). We notice that partially chaotic orbits pch, pchb and phol occupy areas while regular orbits $\mathrm{r} 1, \mathrm{r} 2$ and rhol are curves, the first and the second ones bounding orbit pch and rhol separating pchb from phol. But regular orbit cant is not a continuous but a broken line, i.e. it is a cantori that cannot separate orbits pch and pchb. In fact, we found that the area covered by orbit pchc (not shown) superposes with the areas covered by both pch and pchb, so that these two orbits are in fact a single one. In other words, we have here an example of the phenomenon of stickiness (see, e.g. Contopoulos 2002) where orbit cant (and others like it) presents a barrier to the motion between the regions of phase space covered by orbits pch and pchb, but porous enough to be occasionally traversed. Since the region covered by pch is much larger than that covered by pchb (notice the big difference between the density of points on each area), it is much less likely that the barrier posed by the cantori could be traverse by an orbit with initial conditions in the region of pch than another with initial conditions in the region of pchb. That is probably the reason why orbit pch could not cross that barrier, even after $10^{10}$ iterations, while orbit pchc could. Anyway, it was not an easy task for the latter either, it could do the crossing only after about $3.75 \times 10^{9}$ iterations.

A caveat is necessary here. We should recall that the trajectories obtained for the same chaotic orbit with different hardware or software are very different. In fact, although for regular orbits we obtained essentially the same trajectories with our double and quadruple precision programs that was not the case for the partially chaotic orbits. Therefore, anyone who tries to reproduce our results will find that the orbits we give as regular are regular, and those that we give as partially chaotic are partially chaotic. But it is perfectly possible that, with his computer, he might find that the initial conditions we give for orbit pchb result in an orbit that invades the region covered by our orbit pch, or that the initial conditions for orbit pchc give an orbit that only covers the region of our orbit pchb. However, trying several slightly different initial conditions, he should be able to find orbits that behave as pchb and pchc. 


\section{CONCLUSIONS}

Using a four-dimensional map, we have confirmed all the results obtained by Muzzio (2017) with a toy Hamiltonian model with three degrees of freedom. We found partially chaotic orbits, within a mostly regular domain (see Fig. 2), that occupy a lane part of which is shown in Fig. 3. That lane is made up of curves corresponding to orbits with different values of an integral of motion. Extrapolating those curves we found regular orbits that bound the partially chaotic orbit in question. That is, with a different model we have provided further evidence that partially chaotic orbits exist in cocoons well isolated from the Arnold web by regular orbits. Besides, we found another partially chaotic orbit inside one of the holes of the first one and a regular orbit that separates them. Finally, inside the first partially chaotic orbit, we found a broken regular orbit (a cantori) that poses a porous barrier that hampers the access of that orbit to another partially chaotic domain, i.e. an example of stickiness.

We have emphasized, both in our previous paper and in the present one, that numerical results such as ours are valid only over the time spam covered by the numerical integrations or iterations performed. In that sense, we have here extended the validity of our previous conclusions to an interval 50 times longer, about 50 million Hubble times in a galactic context.

Although the preceding statement is what strict logic dictates, let us speculate in this concluding remarks whether our results can be valid for longer time spans. The big question is: why not? Here, the spectre of Arnold diffusion comes to haunt us, as we know that it is an extremely slow process. But, in that case, there is a good reason for that slowness: the 5D fully chaotic orbits have to find their way through the interstices left by the $3 \mathrm{D}$ regular orbits. Here, instead, we have $4 \mathrm{D}$ partially chaotic orbits surrounded by the 3D tori of regular orbits. How would they escape, no matter how long the time at their disposal? The single answer we can find is that perhaps, somehow, the partially chaotic 4D orbits transform into fully chaotic 5D orbits and then can escape from their 3D prison. But, to that, we can pose another question: is there any mechanism that can make that an orbit that obeys an integral of motion, and for times as long as we have probed, to cease to obey it? Besides, despite the extremely fine grids investigated and the very long integration times (or very large numbers of iterations) used to obtain the LEs of sample orbits, we could find no fully chaotic orbits in the regions of the lanes of partially chaotic orbits investigated by Muzzio (2017) and in this work or their surroundings. Therefore, it seems very unlikely that those partially chaotic orbits might become fully chaotic ones, even for time spans much longer than those covered by our numerical experiments.

The situation is very different in the frontier between the mostly regular central domain and the mostly fully chaotic domain that surrounds it in our Figs 2 and 1 of Muzzio (2017). The structure of that frontier is quite complex, perhaps of a fractal nature and very different from the clear simple lanes studied in both papers, with regular, partially and fully chaotic orbits intermingled. Besides, we have found that is not unusual for partially chaotic orbits in those regions to reveal a fully chaotic nature when the integration of their orbits is pursued for longer times. It is in these regions where one should investigate whether 4D partially chaotic orbits place hurdles to 5D fully chaotic ones but, as already indicated by Muzzio (2017), this will not be an easy task.

Let us finish recalling that partially chaotic orbits are nothing mysterious. They are, in fact, the single chaotic orbits present in Hamiltonian systems with three degrees of freedom that have an additional integral besides energy, e.g. in systems with rotational symmetry that conserve the angular momentum component parallel to the axis of symmetry. Of course, one can argue that those cases can be reduced to systems with two degrees of symmetry, e.g. studying the motion in the meridional plane in systems with rotational symmetry. But, then, does it not happen the same with our orbits? Although we do not know which is the integral that they obey, the 3D Poincaré maps that we obtained are very similar to the usual Poincaré maps for Hamiltonian systems with two degrees of freedom and, besides, we have found that the phenomenon of stickiness is also present here. We leave the question open, but we plan to continue investigating this very interesting problem.

\section{ACKNOWLEDGEMENTS}

We are very grateful to D. Pfenniger for the use of his code, and to H.R. Viturro for his assistance. The comments of an anonymous reviewer were very useful to improve the original version of this paper and are gratefully acknowledged. This work was supported with grants from the Consejo Nacional de Investigaciones Científicas y Técnicas de la República Argentina, the Agencia Nacional de Promoción Científica y Tecnológica and the Universidad Nacional de La Plata.

\section{REFERENCES}

Carpintero D. D., Muzzio J. C., 2016, MNRAS, 459, 1082

Contopoulos G., 1971, AJ, 76, 147

Contopoulos G., 2002, Order and Chaos in Dynamical Astronomy. SpringerVerlag, Berlin

Contopoulos G., Harsoula M., 2010a, Int. J. Bifurcation Chaos, 20, 2005

Contopoulos G., Harsoula M., 2010b, Celest. Mech. Dyn. Astron., 107, 77

Contopoulos G., Harsoula M., 2013, MNRAS, 436, 1201

Contopoulos G., Galgani L., Giorgilli A., 1978, Phys. Rev. A, 18, 1183

Efthymiopoulos C., Harsoula M., 2013, Physica D, 251, 19

Froeschlé C., 1970, A\&A, 4, 115

Froeschlé C., 1971, Ap\&SS, 14, 110

Froeschlé C., 1972, A\&A, 16, 172

Froeschlé C., Scheidecker J.-P., 1973a, A\&A, 22, 431

Froeschlé C., Scheidecker J.-P., 1973b, Ap\&SS, 25, 373

Goodman J., Schwarzschild M., 1981, ApJ, 245, 1087

Harsoula M., Kalapotharakos C., 2009, MNRAS, 394, 1605

Karney C. F. F., 1983, Physica D, 8, 360

Katsanikas M., Patsis P. A., Contopoulos G., 2013, Int. J. Bifurcation Chaos, 23, 1330005

Lichtenberg A. J., Lieberman M. A., 1992, Regular and Chaotic Dynamics, 2nd edn. Springer-Verlag, New York

Meiss J. D., Cary J. R., Grebogi C., Crawford J. L., Kaufman A. N., Abarbanel H. D. I., 1983, Physica D, 6, 375

Merritt D., Valluri M., 1996, ApJ, 471, 82

Milani A., Nobili A. M., 1992, Nature, 357, 569

Milani A., Nobili A. M., Knežević Z., 1997, Icarus, 125, 13

Muzzio J. C., 2003, Bol. Asoc. Argentina Astron., 45, 69

Muzzio J. C., 2017, MNRAS, 471, 4099

Muzzio J. C., Carpintero D. D., Wachlin F. C., 2005, Celest. Mech. Dyn. Astron., 91, 173

Patsis P., Zachilas L., 1994, Int. J. Bifurcation Chaos, 4, 1399

Pettini M., Vulpiani A., 1984, Phys. Lett. A, 106, 207

Richter M., Lange S., Bäcker A., Ketzmerick R., 2014, Phys. Rev. E, 89, 22902

Shirts R. B., Reinhardt W. P., 1982, J. Chem. Phys., 77, 5204

Udry S., Pfenniger D., 1988, A\&A, 198, 135

Voglis N., Contopoulos G., Efthymiopoulos C., 1998, Phys. Rev. E, 57, 372

Zorzi A. F., Muzzio J. C., 2012, MNRAS, 423, 1955

This paper has been typeset from a $\mathrm{T}_{\mathrm{E}} \mathrm{X} / \mathrm{LAT}_{\mathrm{E}} \mathrm{X}$ file prepared by the author. 